Asian Spine Journal

Vol. 1, No. 1, pp 53 56, 2007

\title{
New Cervical Laminoplasty Polyethererketone Cage - Two Case Reports -
}

\author{
Jae-Yoon Chung, Jae-Joon Lee, Jong-Seon Kim, Hyoung-Yeon Seo \\ Department of Orthopedic Surgery, Chonnam National University Hospital, Gwangju, Korea
}

Cervical laminoplasty is a widely used technique to enlarge the spinal canal for spinal cord decompression. The two common methods are an open door laminoplasty and a midline splitting laminoplasty. Several devices and materials have been used as a spacer for maintaining or stabilizing the lamina in the open or split position, however, some are difficult to implant. Moreover, they are not designed to restore the natural dynamics of the cervical spine, and can cause discomfort to the patient. There is a need to develop a device and material that will be effective in maintaining and stabilizing the position of the lamina after laminoplasty, being able to be implanted easily and safely to restore the natural dynamics of the cervical spine, as well as getting the bony union to the host bone. We report two cervical laminoplasty patients who were treated using a new laminoplasty polyetheretherketone cage.

Key Words: Cervical laminoplasty, Polyetheretherketone cage

\section{Introduction}

Cervical laminoplasty is a popular technique for treating multilevel myelopathy or myeloradiculopathy resulting from the condition of the spinal canal stenosis, such as, ossification of the posterior longitudinal ligament (OPLL) or spondylotic change. The two most common methods are spinous process-splitting laminoplasty and open door technique. In a midline splitting laminoplasty, facilitating the reconstruction of posterior neural arch is one of the reported advantages. Until now, several devices and materials have been used as a spacer for maintaining or stabilizing the lamina in the split position. These include hydroxyapatite, xenograft, allograft, autograft, and metal. Some of them have problems in use, and also those are not designed to restore the natural dynamics of the cervical spine (flexionextension), and can cause discomfort to the patient.

This paper reports the results of the two cases which were treated using the new laminoplasty polyetheretherketone (PEEK) cage designed to overcome those pitfalls.

\section{Case Reports}

\section{Case 1}

A 74-year-old male visited our department complaining of clumsiness in both hands and motor weakness in lower extremities that had begun insidiously 2 years earlier. His motor grades were good on both hip flexors and knee extensors but poor on ankle dorsiflexors. Both the soles and calves showed numbness. The patellar jerk increased to $3+$ on the right. The finger escape sign was positive on the right. In addition, the reverse radial reflex, grip and release test were positive. The Motor Index (MI) score was 92 points and the Japanese Orthopaedic Association (JOA) score was 14 points. The plain radiograph showed disc space narrowing from $\mathrm{C} 4$ to $\mathrm{C} 7$ and spondylotic changes with an average Pavlov ratio of 0.69 (0.64 0.74). In addition, the MRI revealed cord signal changes, and the average cord compression for levels C3-C7 was 0.46 (0.44 0.49). A midline splitting laminoplasty was performed using the new

Corresponding author: Jae-Yoon Chung, MD

Department of Orthopedic Surgery, Chonnam National University Hospital

8 Hakdong, Gwangju, 501-757, Korea

Tel: +82-62-227-1640, Fax: +82-62-225-7794, E-mail: jychung@chonnam.ac.kr 
PEEK cage at $\mathrm{C} 3$ and $\mathrm{C} 4$, and using the xenograft at $\mathrm{C} 5$ to C7. The PEEK cage was inserted packed with morcellised bone chip gathered from the tip of spinous process. When using xenograft, the graft material was reshaped to trapezoidal contour like as that of the cage. A small hole was made at the base of split laminae to allow passage of two non-absorbable sutures from the xenograft or cage at each level (Fig. 1A). The graft or cage was sutured tightly with the lamina by diagonal directions (Fig. 1B). Postoperatively, the patient wore a soft collar for one month. The 2 and half years follow-up plain radiograph and computed tomography (CT) at 3 months after surgery showed good maintenance of the lamina and bony union (Fig. 2). The MI score improved to 99 points and the JOA score improved to 17 points on 3-year-follow up.

\section{Case 2}

A 63-year-old male visited our hospital complaining of quadriparesis after falling from a height four years earlier. He had undergone anterior fusion with a plate and tricortical iliac bone at the C4-5-6 level at other hospital after the fall. His symptoms became aggravated after surgery. He had a spastic gait and upper extremities spasticity. The plain radiograph showed spinal canal stenosis with a Pavlov ratio of $0.44(0.34 \sim 0.60)$ and the average cord compression ratio
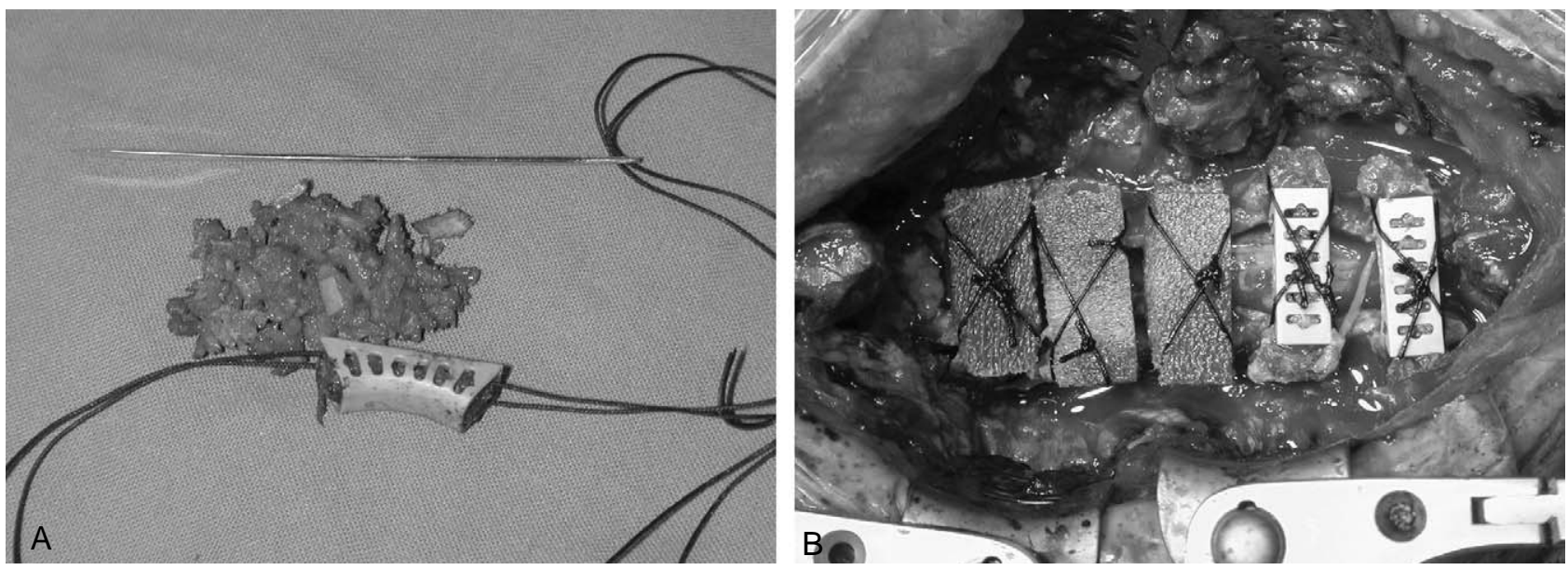

Fig. 1. (A) Laminoplasty Polyethererketone (PEEK) cage packed with morcellised bone graft and connected with non-absorbable sutures. (B) Intraoperative photograph showing a midline splitting laminoplasty using laminoplasty PEEK cage and xenograft.
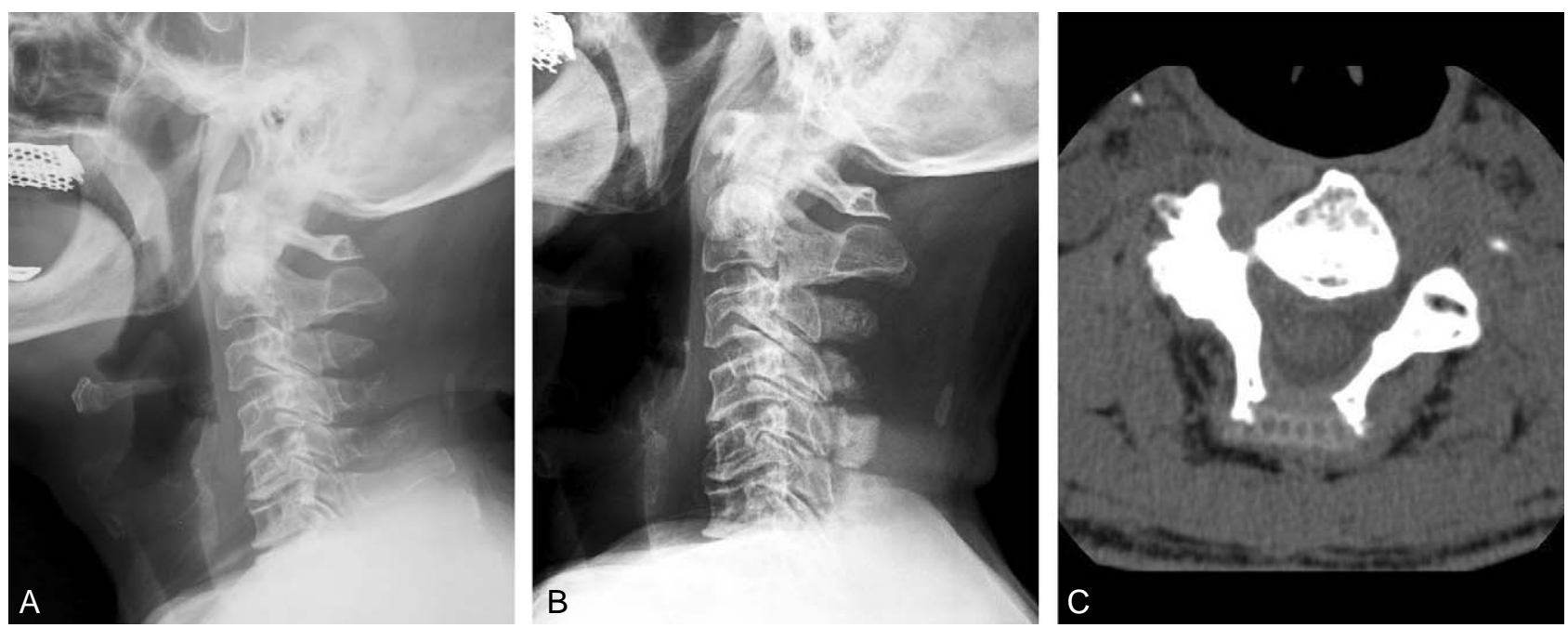

Fig. 2. Preoperative (A) and postoperative 2.5-year radiograph (B) demonstrating canal expansion with the shift of the spinolaminar line in a 74-year-old male (Case 1). The Polyethererketone cage was used as a spacer at C3 and C4 laminoplasty. CT scan at postoperative 3 month $(\mathbf{C})$ showed solid bony union between the cage and spinous process, enlargement of the spinal canal, decompression of the spinal canal, and good muscle reattachment. 
for the $\mathrm{C} 3 \sim \mathrm{C} 7$ levels was 0.39 (0.29 0.54). The preoperative MI and JOA scores were 88 and 11 points, respectively. A midline splitting laminoplasty was performed using the PEEK cage at $\mathrm{C} 3$ and a xenograft at C4-7. Postoperatively, the three months follow up plain radiograph and CT scan showed solid bony union (Fig. 3). Clinically, the MI and JOA scores on 2 years follow up improved to 98 and 17 points, respectively.

\section{Discussion}

In midline splitting laminoplasty, the spinous process was bisected into two halves and rotated outwards. Then a spacer is placed between the halves to secure the opening. Several materials and devices as a spacer have been introduced to maintain and stabilize the lamina in the split state ${ }^{1-5}$. However, they have some problems to implant and do not have a substantial low-profile. In addition, they can potentially cause damage to the surrounding tissue and spinal cord. Accordingly, there is a need for an improved laminoplasty implant that is effective in maintaining and stabilizing the position of the lamina after a laminoplasty. Moreover, there is a need for a device that can be implanted easily and safely, and that will allow for muscle reattachment and restore the natural dynamics of the cervical spine.

The laminoplasty PEEK cage utilized in this study designed by the author (JYC) and obtained a US patent (Laminoplasty cage, 2004, Depuy spine) has a generally hollow, elongated, and arch-shaped ventral surface of the body with open ends (Fig. 1A). So, we can put the bone graft into the cage and also, can enlarge the spinal canal sufficiently. This cage is particularly advantageous in that it is easy to implant, will allow for muscle reattachment, will restore the natural dynamics of cervical spine, and has a substantially low-profile to avoid or prevent potential abrasion or damage to the surrounding tissue. The open ends of the cage are also preferably adapted in order to contour the shape of a bisected spinous process. The anatomical shape of the implant reduces the level of impingement with the adjacent bone structures and grafts, facilitates the secure positioning of the cage, and increases the patient's range of motion. Each side contains slots and perforations except for the dura contact side, which are enough size and shape to allow for the vascularization of the bone graft as well as muscle reattachment.

PEEK used to make a laminoplasty cage is a semicrystalline polyaromatic linear polymer that provides a good combination of strength, stiffness, toughness, and environmental resistance. The elastic modulus of the PEEK cage is close to the bone, approximately $17 \mathrm{Gpa}$, which helps decrease the level of stress shielding ${ }^{6,7}$.

More cases and long-term evaluations will be needed. However, in our cases, the clinical and radiographic outcomes of cervical laminoplasty using the new PEEK laminoplasty cage were satisfactory. Therefore, the PEEK laminoplasty cage is a good substitute for other devices or materials used in midline splitting cervical laminoplasty and
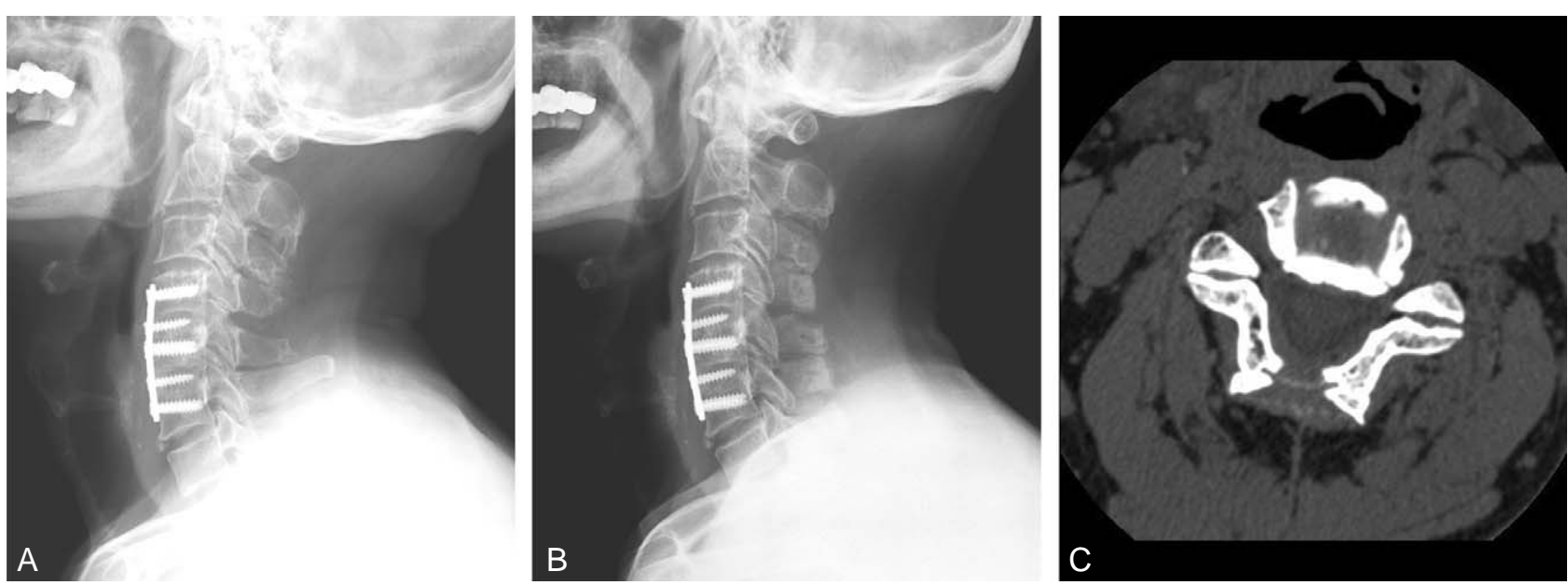

Fig. 3. Preoperative lateral radiograph (A) and postoperative 3-month radiograph (B) of a 64-year-old male (Case 2). The PEEK cage was used at C3 laminoplasty. CT scan (C) taken 3 month after surgery showed that the laminoplasty Polyethererketone cage effectively maintains and stabilizes the position of the lamina and spinal cord decompression. 
possibly open door cervical laminoplasty.

\section{REFERENCES}

1. Chung JY, Kim YW, Joo DC: The results of spinous process-splitting laminoplasty using xenograft spinous process spacer. J Korean Orthop Assoc 2000; 35: 641-645.

2. Kurokawa T, Tsuyama N, Tanaka H, et al: Enlargement of spinal canal by the sagittal splitting of the spinous process. Bessatsu Seikeigeka 1982; 2: 234-240.

3. O'Brien MF, Peterson D, Casey AT, Crockard HA: A novel technique for laminoplasty augmentation of spinal canal area using titanium miniplate stabilization. A computerized morphometric analysis. Spine 1996; 21: 474-483.
4. Park AE, Heller JG: Cervical laminoplasty: use of a novel titanium plate to maintain canal expansion--surgical technique. J Spinal Disord Tech 2004; 17: 265-271.

5. Tomita K, Kawahara N, Toribatake Y, Heller JG: Expansive midline T-saw laminoplasty (modified spinous process-splitting) for the management of cervical myelopathy. Spine 1998; 23: 32-37.

6. Cho DY, Liau WR, Lee WY, Liu JT, Chiu CL, Sheu PC: Preliminary experience using a polyetheretherketone (PEEK) cage in the treatment of cervical disc disease. Neurosurgery 2002; 51: 1343-1349.

7. Jockisch KA, Brown SA, Bauer TW, Merritt K: Biological response to chopped-carbon-fiber-reinforced peek. J Biomed Mater Res 1992; 26: 133-146. 